\title{
Characterisation of phenolic acid derivatives and flavonoids from different morphological parts of Helichrysum obconicum by a RP-HPLC-DAD-(-)-ESI-MS ${ }^{n}$ method
}

\author{
Sandra Gouveia, Paula C. Castilho* \\ Centro de Química da Madeira, CCCEE, Universidade da Madeira, Campus Universitário da Penteada, 9000-390 Funchal, Portugal
}

\section{A R T I C L E I N F O}

\section{Article history:}

Received 14 June 2010

Received in revised form 31 March 2011

Accepted 24 April 2011

Available online 1 May 2011

\section{Keywords:}

Hydroxycinnamic acids

Flavonoids

LC-MS $^{n}$

Helichrysum

\begin{abstract}
A B S T R A C T
The phenolic composition from different morphological parts of Helichrysum obconicum was investigated for the first time and 50 different phenolic compounds were detected. Phenolic acid conjugates, mainly mono- and di-caffeoylquinic acid derivatives, were the major components; some flavonoid derivatives were also detected in small amounts. Their separation and identification was performed by a high-performance liquid chromatography/electron spray ionisation tandem ion trap mass spectrometry method, with special emphasis on $\mathrm{MS}^{n}$ fragmentation. The presence of di- and tricaffeoylshikimic acid isomers in Helichrysum species extracts was reported for the first time, the spectra of these compounds were mainly characterised by the presence of a [caffeoylshikimic acid- $\mathrm{H}]^{-}$ion at $\mathrm{m} / \mathrm{z} 335$. A lamiridosins-di- $\mathrm{O}$-hexoside, an unusual component in Asteraceae species, was also detected.
\end{abstract}

(c) 2011 Elsevier Ltd. All rights reserved.

\section{Introduction}

The vascular flora of Madeira Archipelago is exuberant and diverse, comprising over 1220 species of which $10 \%$ are endemic. Amongst this vast number of plants, a large variety is still used in the local traditional medicine.

Four endemic species of the genus Helichrysum (Asteraceae) are known and the use of the three abundant ones in traditional medicine is well reported (Rivera \& Obón, 1995). One of these endemic species is Helichrysum obconicum DC. E; its aerial parts are used as herbal tea for curing digestive, stomachic and intestinal diseases. Recently, chefs from high class hotels have shown interest of introducing this new flavour as a neutraceutical in herbal teas and salads.

The main classes of compounds usually found in Helichrysum species are phenolic compounds, coumarins and terpenoids (AlRehaily, Albishi, El-Olemy, \& Mossa, 2008).

The phenolic composition of two other Helichrysum species endemic from Madeira has been investigated before by our group and flavonoids and phenolic acid derivatives were found to be the major bioactive constituents (Gouveia \& Castilho, 2009, 2010).

Phenolic compounds are a heterogeneous group of secondary metabolites in vascular plants, vital for normal plant development.

\footnotetext{
* Corresponding author. Address: Centro de Química da Madeira, CCCEE, Universidade da Madeira, Campus Universitário da Penteada, Piso 0, 9000-390 Funchal, Portugal. Tel.: +351 291705102; fax: +351 291705149.

E-mail address: castilho@uma.pt (P.C. Castilho).
}

They are known to be responsible for the colour and flavour of many plants but their main interest relies on their biological properties such as antioxidant activity, protection against cancer, cardiovascular and neurodegenerative diseases.

The main subclasses of phenolic compounds are phenolic acids and flavonoids. The phenolic acids are mostly hydroxybenzoic acids and hydroxycinnamic acids derived from benzoic and cinnamic acids, respectively (Mattila \& Kumpulainen, 2002). Flavonoids normally occur as flavonoid $\mathrm{O}$-glycosides or $\mathrm{C}$-glycosides and, in some cases, additional hydroxylation, acylation and/or methylation occur(s) (Cuyckens \& Claeys, 2004).

The interest in the analysis and identification of the phenolic compounds and their derivatives present in medicinal plant extracts rises not only from the need to find new sources for these compounds but also to establish a relationship between type/ structure of the compounds and the extract uses in traditional medicine.

Reversed phase high-performance liquid chromatography (RPHPLC) hyphenated with mass spectrometry techniques using APCI and ESI interfaces has proved to be a powerful tool for the analysis of plant extract composition. The use of ESI operating in the negative mode $\left(\mathrm{ESI}^{-}\right)$has demonstrated to be more selective and efficient in the characterisation of phenolic compounds even those present in trace amounts (Gouveia \& Castilho, 2009).

The use of combined techniques allowed for the characterisation of 50 compounds in $\mathrm{H}$. obconicum. Most of them are phenolic acid derivatives formed by the esterification of caffeic acid and quinic acid or shikimic acid. They are all described for the first time in this plant. 
In this study, the different morphological parts of the plant used in traditional medicine were analysed in order to establish if there is or not a preferential accumulation of phenolic compounds in one or more parts.

The occurrence of quinic acid derivatives in polar extracts of Helichrysum species was reported before (Gouveia \& Castilho, 2009), (Carini, Aldini, Furlanetto, Stefani, \& Facino, 2001) but not the presence of shikimic acid derivatives.

\section{Experimental}

\subsection{Chemical and standards}

HPLC grade acetonitrile $\left(\mathrm{CH}_{3} \mathrm{CN}\right)$ (Lab-Scan, 99\%; Gliwice, Poland), ultra-pure water (Milli-Q Waters, EUA) and formic acid (analytical grade, Merck, Germany) were used for mobile phase preparation in the LC-MS analysis. The methanol used for extraction of H. obconicum was AR grade, purchased from Fisher (Lisbon, Portugal). Eluents prepared for LC-MS analysis were additionally filtered through $0.45 \mu \mathrm{m}$ Nylon membranes (Millipore).

Reference substances apigenin (>99\%, HPLC), quercetin (>99\%, HPLC) and quercetin-3-O-glucoside were purchased from Extrasynthese (Lyon, France) and 5-O-caffeoylquinic acid, kaempferol from Acros Organics (Geel, Belgium). 1,3-O-dicaffeoylquinic acid, 1,5-O-dicaffeoylquinic acid, 3,4-O-dicaffeoylquinic acid, 3,5-O-dicaffeoylquinic acid, 3,5-O-dicaffeoylquinic acid and 3,4,5-O-tricaffeoylquinic acid were obtained from Chengdo biopurity phytochemicals, Lts China (Sichuan, China).

Stock solutions of these compounds $(100 \mu \mathrm{g} / \mathrm{mL})$ were prepared in ethanol and stored in a refrigerator at $-20^{\circ} \mathrm{C}$ until us for further analys by HPLC-DAD-ESI-MS ${ }^{n}$.

\subsection{Plant material and sample preparation}

Samples of $H$. obconicum were collected by us from the wild in the North Coast of Madeira Island. The collected plant material consisted of total aerial parts and part of it was separated into leaves, flowers and stems for individual analysis. However, the amount of collected flowers was not sufficient to be analysed. The plants were authenticated by taxonomist Fátima Rocha and a voucher was deposited in the Madeira Botanical Garden herbarium collection.

Dried and powdered plant material (100 g) was exhaustively extracted by maceration with methanol $(1 \mathrm{~L})$, at room temperature for $24 \mathrm{~h}$.

In all cases, the solutions were filtered and concentrated to dryness under reduced pressure in a rotary evaporator $\left(<40^{\circ} \mathrm{C}\right)$. Stock solutions with concentrations ( $\mathrm{m} / \mathrm{v}$ ) of $5 \mathrm{mg} / \mathrm{mL}$ were prepared by dissolving dried extract in initial HPLC mobile phase aqueous formic acid $0.1 \%(\mathrm{v} / \mathrm{v})-\mathrm{CH}_{3} \mathrm{CN}(20: 80, \mathrm{v} / \mathrm{v})$.

These solutions were filtered through $0.45 \mu \mathrm{m}$ Nylon micropore membranes prior to use and $10 \mu \mathrm{L}$ were injected for HPLC-DADESI-MS $^{n}$ analysis. Three independent assays were performed for each sample.

\subsection{HPLC-DAD-ESI-MS ${ }^{n}$ analysis}

\subsubsection{Liquid chromatography}

The HPLC system consisted of a Dionex ultimate 3000 series instrument (California, EUA) coupled to a binary pump, a diodearray detector (DAD), an autosampler and a column compartment. UV-visible spectra were recorded from 210 to $520 \mathrm{~nm}$ and chromatograms were monitored at $280 \mathrm{~nm}$. Samples were separated on a Phenomenex Gemini $C_{18}$ column $(5 \mu \mathrm{m}, 250 \times 3.0 \mathrm{~mm}$ i.d., Phenomenex) with a sample injection volume of $10 \mu \mathrm{L}$. The mobile phase consisted of acetonitrile (A) and water-formic acid (100:0.1, $\mathrm{v} / \mathrm{v})(\mathrm{B})$. The eluting conditions applied were: 0-10 min, linear gradient from $20 \%$ to $25 \% \mathrm{~A}$; $10-20 \mathrm{~min}, 25 \%$ A isocratic; $20-40 \mathrm{~min}$, linear gradient from $25 \%$ to $50 \% \mathrm{~A} ; 42-47 \mathrm{~min}$, linear gradient from $50 \%$ to $100 \%$ A for washing, return to $20 \%$ A at 55 min, and finally $5 \mathrm{~min}$ isocratic to re-equilibrate the column. The mobile phase flow rate was $0.4 \mathrm{~mL} / \mathrm{min}$ and column temperature was controlled at $30^{\circ} \mathrm{C}$.

\subsubsection{Mass spectrometry}

The HPLC system described above was coupled on-line to a Bruker Esquire (Bremen, Germany) model 6000 ion trap mass spectrometer fitted with an ESI source. Data acquisition and processing were performed using Esquire control software. Negative ion mass spectra of the column eluate were recorded in the range $m / z$ $100-1000$ at a scan speed of $13000 \mathrm{Da} / \mathrm{s}$. High purity nitrogen $\left(\mathrm{N}_{2}\right)$ was used both as drying gas at a flow rate of $10.0 \mathrm{~mL} / \mathrm{min}$ and as a nebulising gas at a pressure of $50 \mathrm{psi}$. The nebuliser temperature was set at $365^{\circ} \mathrm{C}$ and a potential of $+4500 \mathrm{~V}$ was used on the capillary. Ultrahigh-purity helium ( $\mathrm{He}$ ) was used as collision gas at a pressure of $1 \times 10^{-5}$ mbar and the collision energy was set at $40 \mathrm{~V}$.

The acquisition of $\mathrm{MS}^{n}$ data was made with auto $M S^{n}$ mode, with isolation width of $4.0 \mathrm{~m} / z$. For $\mathrm{MS}^{n}$ analysis, mass spectrometer was scanned from 10 to $1000 \mathrm{~m} / z$ with fragmentation amplitude of $1.0 \mathrm{~V}\left(\mathrm{MS}^{n}\right.$ up to $\left.\mathrm{MS}^{4}\right)$ and two precursor ions.

\section{Results and discussion}

The HPLC base peak chromatograms (BPC) in the negative mode profiles of the $H$. obconicum total aerial parts, leaves and stems methanolic extracts are presented in Fig. 1.

The HPLC conditions used allowed for a good separation of a large percentage of compounds and no variation was observed in the three analysis of each sample.

Compounds were characterised based on their HPLC retention time, UV spectra and mostly on their MS ${ }^{n}$ fragmentation behaviour due to the lack of reference standards for the majority of the extract components.

Most of the detected compounds showed similar UV absorptions maxima with two bands at $230-240 \mathrm{~nm}$ and $320-330 \mathrm{~nm}$ and a shoulder at $290-300 \mathrm{~nm}$. This type of UV spectra is characteristic of hydroxycinnamic acids. Some peaks with characteristic UV absorptions band for flavonoids were also detected (Mabry, Markham, \& Thomas, 1970).

The first approach to the $\mathrm{MS}^{1}$ spectrum was to identify the deprotonated molecular ion $[\mathrm{M}-\mathrm{H}]^{-}$. Generally, for the detected phenolic compounds, the $[\mathrm{M}-\mathrm{H}]^{-}$ion corresponds to the most intense peak in the $\mathrm{MS}^{1}$ spectra.

Whenever isomers already found on endemic Helichrysum species of Madeira Archipelago were detected, their differentiation was achieved based on previous reports where the same analytical method was applied. The next subsections present the description of the characterisation of the observed compounds which were grouped in two types: phenolic acids and flavonoids. The compounds were numbered by their order of elution.

The main fragments observed in the $\mathrm{MS}^{n}$ experiments $(n=1-4)$ are given in Table 1 and the structures of the compounds found in H. obconicum are shown in Fig. 2.

\subsection{Phenolic acid derivatives}

Amongst the components of $H$. obconicum extracts, there were several phenolic acids derivatives such as the esters of caffeic and quinic or shikimic acids. For the majority of the detected compounds the deprotonated molecular ion, $[\mathrm{M}-\mathrm{H}]^{-}$, had 


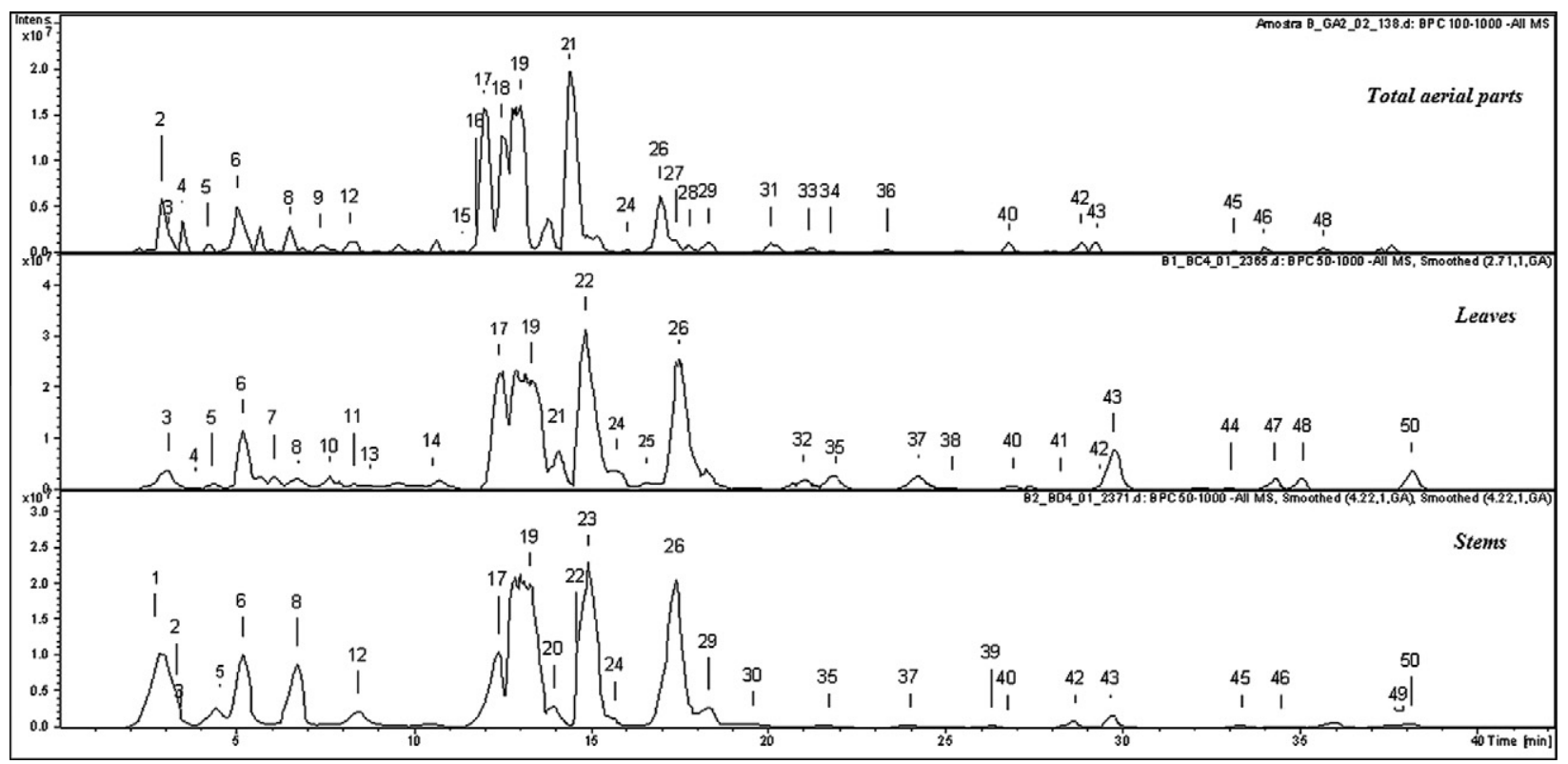

Fig. 1. HPLC-DAD-ESI-MS ${ }^{n}$ chromatograms (Base Peak Chromatograms - BPC) of Helichrysum obconicum methanolic extracts: total aerial parts, leaves and stems.

sufficient intensity to be submitted to subsequent $\mathrm{MS}^{n}$ fragmentation. The loss of the substitution groups are referred to the $[\mathrm{M}-\mathrm{H}]^{-}$ ion.

\subsubsection{Quinic acid}

The presence of quinic acid derivatives on plants from the Helichrysum genus has been already reported (Gouveia \& Castilho, 2009).

Compound $3\left(t_{R}=3.1 \mathrm{~min}\right)$ showed a $[\mathrm{M}-\mathrm{H}]^{-}$ion at $\mathrm{m} / z 191$ and its $\mathrm{MS}^{2}$ fragmentation gave a fragment ion at $m / z 127$ characteristic of quinic acid.

\subsubsection{Caffeoylquinic acid}

$\mathrm{MS}^{n}$ fragmentation experiments of caffeoylquinic acid isomers are well studied and the locations of the caffeoyl groups in the quinic acid structure can be determined taking into account the relative intensities of characteristics $\mathrm{MS}^{n}$ ions (Clifford, Knight, \& Kuhnert, 2005).

Two compounds $5\left(t_{R}=4.3 \mathrm{~min}\right)$ and $6\left(t_{R}=5.0 \mathrm{~min}\right)$ gave a $[\mathrm{M}-\mathrm{H}]^{-}$ion at $\mathrm{m} / \mathrm{z} 353$ and were detected in all extracts. The $\mathrm{MS}^{2}$ spectrum of compound $5\left(t_{R}=4.3 \mathrm{~min}\right)$ showed a fragment ion at $m / z 191$ as base peak and an intense ion at $m / z 179$ (>40\% of base peak). Based on the hierarchical key proposed by Clifford et al. (2005) compound 5 was characterised as 3-O-caffeoylquinic acid.

Compound 6 was identified as 5-O-caffeoylquinic acid by comparing its UV and MS ${ }^{n}$ spectra and HPLC retention time with those of a reference standard.

Besides the monocaffeoylquinic acids isomers, there are six dicaffeoylquinic acid derivatives in $H$. obconicum.

Compounds $8 \quad\left(t_{R}=6.6 \mathrm{~min}\right), \quad \mathbf{1 7} \quad\left(t_{R}=12.1 \mathrm{~min}\right), \quad \mathbf{1 8}$ $\left(t_{R}=12.5 \mathrm{~min}\right), 19\left(t_{R}=13.2 \mathrm{~min}\right), 22\left(t_{R}=14.7 \mathrm{~min}\right)$ and $31\left(t_{R}=\right.$ $20.1 \mathrm{~min})$ all exhibited $[\mathrm{M}-\mathrm{H}]^{-}$ions at $\mathrm{m} / z 515$. MS $\mathrm{S}^{2}$ fragmentation of the ion at $m / z 515$ gave a fragment ion at $m / z 353$, as base peak, suggesting the loss of a caffeoyl residue (162 Da).

They were identified by comparison with standard compounds as 1,3-O-dicaffeoylquinic acid (compound 8), 3,4-O-dicaffeoylquinic acid (compound 17), 1,5-O-dicaffeoylquinic acid (compound 18), 3,5-O-dicaffeoylquinic acid (compound 19) and 4,5-O-dicaffeoylquinic acid (compound 22).
The occurrence of dicaffeoylquinic acid isomers $8,17,18$ and 19 in Helichrysum species has been reported before, namely in our recent work on endemic Helichrysum species from Madeira Archipelago where the completely fragmentation characterisation is presented (Gouveia \& Castilho, 2009).

Compound 31, which is a 4-OH substituted quinic acid $\left(\mathrm{MS}^{3}\right.$ spectrum base peak at $m / z$ 173) showed a $\mathrm{MS}^{2}$ ion at $m / z 299$ and was identified as 1,4-O-dicaffeoylquinic acid (Clifford et al., 2005).

Two compounds $13\left(t_{R}=8.8 \mathrm{~min}\right)$ and $43\left(t_{R}=29.7 \mathrm{~min}\right)$ displayed the same deprotonated molecular ion at $\mathrm{m} / \mathrm{z} 677$.

Compound $\mathbf{4 3}$ was found in all morphological parts of the plant and its $\mathrm{MS}^{n}$ fragmentation showed three consecutive losses of caffeoyl moieties (162 Da) which are consistent with those found for a standard solution of 3,4,5-tricaffeoylquinic acid and comparing to literature reports (Gouveia \& Castilho, 2009).

Despite the fact that compound $\mathbf{1 3}$ gave the same $[\mathrm{M}-\mathrm{H}]^{-}$ion as compound $\mathbf{4 3}$, it occurred at a much shorter retention time. In the $\mathrm{MS}^{2}$ spectrum, a neutral loss of 162 Da was also observed forming a fragment ion at $m / z 515$. This 162 Da residue can be associated to a hexoside group rather than to a caffeoyl group, justifying the low retention time of this compound. The presence of a $\mathrm{MS}^{3}$ ion at $m / z 335$ ( $>30 \%$ of base peak) and a strong $\mathrm{MS}^{4}$ ion at $\mathrm{m} / \mathrm{z} 179$ ( $>80 \%$ of base peak) indicates a $1,3-O$-dicaffeoylquinic acid hexoside.

The hexoside group must be linked to one of the caffeoyl groups since an intense fragment ion [caffeic acid + hexoside- $\mathrm{H}]^{-}$at $\mathrm{m} / \mathrm{z}$ 341 was observed. However, it was not possible to identify which of the caffeoyl group is glycosylated. Thus, compound $\mathbf{1 3}$ was assigned as 1,3-O-dicaffeoylquinic acid hexoside.

Compound $42\left(t_{R}=29.1 \mathrm{~min}\right)$ showed a $[\mathrm{M}-\mathrm{H}]^{-}$ion at $m / z 557$ and was found in all analysed extracts. Its $\mathrm{MS}^{2}$ spectrum gave a fragment ion at $m / z 395$ due to the loss of a 162 Da residue. Fragmentation of the $\mathrm{MS}^{2}$ ion at $\mathrm{m} / \mathrm{z} 395$ gave a fragment ion at $\mathrm{m} / \mathrm{z}$ 233 , reported before as being acetylquinic acid (Gouveia \& Castilho, 2009). These two successive losses of $162 \mathrm{Da}$ and the presence of the ion at $\mathrm{m} / \mathrm{z} 233$ indicate a dicaffeoylquinic acid structure substituted with an acyl group. The loss of a caffeoyl-acyl residue was not observed, so it is possible to deduce that the acyl group is directly connected to the quinic acid structure. However, with 


\begin{tabular}{|c|c|c|c|c|}
\hline No. & $t_{R}(\min )$ & $\mathrm{UV} \lambda_{\max }(\mathrm{nm})$ & {$[\mathrm{M}-\mathrm{H}]^{-} m / z$} & HPLC-DAD-ESI-MS ${ }^{n} m / z$ (\% base peak) \\
\hline 1 & 2.7 & $225,276,300$ & 487 & $\begin{array}{l}\mathrm{MS}^{2} \text { [487]: } 341(100), 251(10.0), 179(52.2), 145(98.6) \\
\mathrm{MS}^{3}[487 \rightarrow 341]: 179(100), 161(11.9), 143(24.0), 113(16.7), 101(16.6) \\
\mathrm{MS}^{4}[487 \rightarrow 341 \rightarrow 179]: 118(32.0), 111(100), 89(72.6)\end{array}$ \\
\hline 2 & 2.8 & - & 683 & $\begin{array}{l}\mathrm{MS}^{2} \text { [683]: } 342(10.8), 341(100) \\
\mathrm{MS}^{3}[683 \rightarrow 341]: 179(100), 161(14.2), 143(18.3), 119(20.4), 113(20.3) \\
\mathrm{MS}^{4}[683 \rightarrow 341 \rightarrow 179]: 119(60.3), 113(32.7), 106(100), 101(45)\end{array}$ \\
\hline 3 & 3.1 & - & 191 & $\begin{array}{l}\mathrm{MS}^{2} \text { [191]: } 173 \text { (69.4), } 171(32.7), 155(35.2), 127 \text { (100), } 111 \text { (34.5), } 109 \text { (30.8 } \\
\text { MS }^{3}[191 \rightarrow 127]: 110(33.6), 109(100)\end{array}$ \\
\hline 4 & 3.5 & 254,292 & 317 & $\begin{array}{l}\mathrm{MS}^{2} \text { [317]: } 225 \text { (100), } 207(13.1), 165(31.5), 125(25.8) \\
\left.\mathrm{MS}^{3} \text { [317 } \rightarrow 225\right]: 207(81.5), 125(91.2), 165(100)\end{array}$ \\
\hline 5 & 4.2 & $243,296,324$ & 353 & $\begin{array}{l}\mathrm{MS}^{2} \text { [353]: } 191(100), 179(37.8), 173(2.7), 135(13.9) \\
\mathrm{MS}^{3}[353 \rightarrow 191]: 173(51.7), 127(100), 111(40.5), 109(25.1) \\
\mathrm{MS}^{4}[353 \rightarrow 191 \rightarrow 127]: 109(100)\end{array}$ \\
\hline $6^{*}$ & 5.0 & $242,300,325$ & 353 & $\begin{array}{l}\mathrm{MS}^{2} \text { [353]: } 191(100), 179(3.5), 135(1.7) \\
\mathrm{MS}^{3}[353 \rightarrow 191]: 173(53.7), 127(100), 111(23.6), 109(20.7) \\
\text { MS }^{4}[353 \rightarrow 191 \rightarrow 127]: 109(100)\end{array}$ \\
\hline 7 & 6.0 & 272,354 & 609 & $\begin{array}{l}\mathrm{MS}^{2} \text { [609]: } 447(31.7), 429(7.81 \%), 285(100), 283(29.6), 257(46.8) \\
\mathrm{MS}^{3}[609 \rightarrow 285]: 257(46.8), 255(100), 219(15.8), 213(95.5), 163(56.0) \\
\mathrm{MS}^{4}[609 \rightarrow 285 \rightarrow 255]: 135(100)\end{array}$ \\
\hline $8^{*}$ & 6.5 & $232,303,321$ & 515 & $\begin{array}{l}\mathrm{MS}^{2} \text { [515]: } 353(100), 335(33.8), 191(24.6), 179(33.9) \\
\mathrm{MS}^{3}[515 \rightarrow 353]: 191(100), 179(45.2), 135(17.4) \\
\mathrm{MS}^{4}[515 \rightarrow 353 \rightarrow 191]: 173(64.9), 127(100), 111(50.0), 109(26.0)\end{array}$ \\
\hline 9 & 7.3 & - & 533 & $\begin{array}{l}\mathrm{MS}^{2} \text { [533]: } 515(11.2), 371(100), 353(21.3), 335(4.5), 173(5.5) \\
\mathrm{MS}^{3}[533 \rightarrow 371]: 353(100), 191(40.7), 179(24.6), 173(33.7) \\
\mathrm{MS}^{4}[533 \rightarrow 371 \rightarrow 353]: 191(100), 179(38.5), 173(18.4), 135(12.2)\end{array}$ \\
\hline 10 & 7.7 & 216,320 & 533 & $\begin{array}{l}\mathrm{MS}^{2}[533]: 372(16.4), 371(100), 353(17.0) \\
\mathrm{MS}^{3}[533 \rightarrow 371]: 353(100), 191(45.4), 173(53.4), 135(76.9) \\
\operatorname{MS}^{4}[533 \rightarrow 371 \rightarrow 353]: 191(96.8), 179(100), 173(68.6), 135(12.2)\end{array}$ \\
\hline 11 & 8.1 & 306 & 337 & $\begin{array}{l}\operatorname{MS}^{2}[337]: 191(100) \\
\operatorname{MS}^{3}[337 \rightarrow 191]: 173(33.2), 127(100), 125 \text { (72.6), } 110(87.0), 93(55.4)\end{array}$ \\
\hline 12 & 8.4 & 232,280 & 567 & $\begin{array}{l}\mathrm{MS}^{2}[567]: 342(22.2), 341(100), 330(31.9), 329(88.2) \\
\operatorname{MS}^{3}[567 \rightarrow 341]: 327(12.9), 326(100), 311(13.8) \\
\operatorname{MS}^{4}[567 \rightarrow 341 \rightarrow 327]: 311(100)\end{array}$ \\
\hline 13 & 8.8 & 255,318 & 677 & $\begin{array}{l}\mathrm{MS}^{2} \text { [677]: } 516(21.5), 515(100), 353(15.6) \\
\mathrm{MS}^{3}[677 \rightarrow 515]: 353(100), 341(54.0), 335(37.4), 323(17.8), 179(80.2) \\
\operatorname{MS}^{4}[677 \rightarrow 515 \rightarrow 353]: 191(100), 179(86.5)\end{array}$ \\
\hline $14^{*}$ & 10.1 & 258,353 & 463 & $\begin{array}{l}\mathrm{MS}^{2} \text { [463]: } 301(100), 300(19.4) \\
\left.\mathrm{MS}^{3} \text { [463 } \rightarrow 301\right]: 271(49.2), 255(20.8), 179(100), 175(17.0), 151(62.7) \\
\mathrm{MS}^{4}[463 \rightarrow 301 \rightarrow 179]: 169(100), 151(94.8)\end{array}$ \\
\hline 15 & 11.4 & - & 415 & $\begin{array}{l}\operatorname{MS}^{2} \text { [415]: } 371(5.6), 179(100), 161(29.0), 143(12.1) \\
\left.\operatorname{MS}^{3} \text { [415 } \rightarrow 179\right]: 143(59.5), 119(100), 113(58.0)\end{array}$ \\
\hline 16 & 11.7 & 262,351 & 451 & $\begin{array}{l}\mathrm{MS}^{2}[451]: 244(16.2), 243(100), 199(1.8) \\
\mathrm{MS}^{3}[451 \rightarrow 243]: 211(53.8), 199(100), 143(89.5), 123(36.1) \\
\mathrm{MS}^{4}[451 \rightarrow 243 \rightarrow 199]: 184(100)\end{array}$ \\
\hline $17^{*}$ & 12.1 & $243,300,324$ & 515 & $\begin{array}{l}\mathrm{MS}^{2}[515]: 353(100), 335(8.6), 179(32.2), 173(39.5) \\
\mathrm{MS}^{3}[515 \rightarrow 353]: 191(41.7), 179(48.0), 173(100), 135(8.4) \\
\mathrm{MS}^{4}[515 \rightarrow 353 \rightarrow 173]: 155(75.3), 111(100), 109(31.0)\end{array}$ \\
\hline
\end{tabular}
Morphological part

Caffeic acid-O-hexosid

Stems

Dimer of caffeic acid-O-hexosie

Total aerial parts

Stems

Quinic acid

Total aerial parts

Leaves

Stems

Unknown

Total aerial parts

Leaves

3-O-Caffeoylquinic acid

Total aerial parts

Leaves

Stems

5-O-Caffeoylquinic acid

Total aerial parts

Leaves

Stems

Kaempferol-di-O-hexoside

Leaves

1,3-O-Dicaffeoylquinic acid

Total aerial parts

Leaves

Stems

Caffeic acid-O-hexoside derivative

Total aerial parts

Caffeic acid-O-hexoside derivative

Leaves

5-O-p-Coumaroylquinic acid

Leaves

Dimethoxylflavanone derivative

Total aerial parts Stems

1,3-O-Dicaffeoylquinic acid hexoside

Leaves

Quercetin-3-O-glucoside.

Leaves

Caffeic acide derivative

Total aerial parts

Unknown

Total aerial parts

3,4-0-Dicaffeoylquinic acid

Total aerial parts

Leaves

Stems 
$\mathrm{MS}^{2}[515]: 353(100), 191(119)$

MS $^{3}$ [515 $\left.\rightarrow 353\right): 191(100), 179(3)$

$\mathrm{MS}^{3}$ [515 $\rightarrow$ 353]: 191 (100), 179 (39.6), 135 (7.8)

$\begin{array}{llll}20 & 13.9 & 244,297,326 & 601\end{array}$

$21 \quad 14.5 \quad 243,299,326 \quad 601$

$22^{*} \quad 14.7-245,297,327 \quad 515$

$23 \quad 15.0-601$

$24 \quad 15.7 \quad 266,332 \quad 445$

$25 \quad 16.6$
MS 2 [601]: 557 (30.7), 516 (39.8), 515 (95.5), 439 (15.3), 395 (100), 377 (21.8) $\mathrm{MS}^{3}$ [601 $\rightarrow$ 395]: 335 (48.4), $233(100), 179(51.8), 173(58.1)$ $\mathrm{MS}^{4}[601 \rightarrow 395 \rightarrow 233]: 173(100)$

$\mathrm{MS}^{2}$ [515]: 353 (100), 179 (10.0), 173 (28.2)

$\mathrm{MS}^{3}[515 \rightarrow 353]: 191(23.7), 179$ (67.7), $173(100), 135$ (11.3) $\mathrm{MS}^{4}[515 \rightarrow 353 \rightarrow 173]: 155$ (43.8), 111 (100)

MS $^{2}$ [515]: 353 (100), 299 (11.5), 203 (20.4), 179 (19.9), 173 (22.8) $\mathrm{MS}^{3}$ [515 $\rightarrow 353$ ]: 191 (20.6), $179(53.9), 173$ (100), 135 (10.3) $\mathrm{MS}^{4}$ [515 $\left.\rightarrow 353 \rightarrow 191\right]$ : 127 (14.0), 109 (12.3), 93 (100)

$\mathrm{MS}^{2}$ [601]: 557 (62.5), 515 (100), 395 (82.3), 233 (32.4)

$\mathrm{MS}^{3}$ [601 $\rightarrow$ 515]: 354 (13.4), 353 (100), 335 (14.1), 191 (82.1)

$\mathrm{MS}^{4}[601 \rightarrow 515 \rightarrow 353]: 192(16.9), 191(100)$

$\mathrm{MS}^{2}$ [455]: 281 (31.7), 270 (20.9), 269 (100), 175 (12.3

MS $^{3}$ [455 $\rightarrow 269$ ]: 225 (92.3), 224 (23.3), 201 (26.6), 151 (42.5), 149 (100)

$\mathrm{MS}^{4}$ [445 $\left.\rightarrow 269 \rightarrow 149\right]: 107(100)$

MS$^{2}$ [499]: 353 (100), 337 (27.1), 335 (37.2), 319 (28.6), 179 (22.1), 173 (61.8)

$\mathrm{MS}^{3}$ [499 $\left.\rightarrow 353\right]: 191$ (73.9), $179(51.0), 173(100), 135$ (30.1)

$\mathrm{MS}^{4}$ [499 $\left.\rightarrow 353 \rightarrow 173\right]: 111(100), 93$ (58.7)

$\mathrm{MS}^{2}$ [601]: 557 (50.9), 395 (100)

$\mathrm{MS}^{3}$ [601 $\rightarrow$ 395]: 233 (100), $173(78.3)$

MS $^{4}$ [601 $\left.\rightarrow 395 \rightarrow 233\right]: 173(100)$

MS $^{2}$ [499]: 338 (13.6), 337 (100), 335 (6.8), 173 (14.4), 163 (24.0)

$\mathrm{MS}^{3}$ [499 $\rightarrow$ 337]: 191 (6.3), 173 (44.8), 163 (100), 119 (10.1)

MS $^{4}$ [499 $\rightarrow 337 \rightarrow 163$ ]: 120 (5.7), 119 (100), 118 (4.8)

$\mathrm{MS}^{2}$ [499]: 354 (17.2), 353 (100), 337 (9.9), 191 (4.6), 179 (3.7)

$\mathrm{MS}^{3}$ [499 $\rightarrow$ 353]: 191 (100), 179 (47.1), 173 (3.5), 135 (14.5)

$\mathrm{MS}^{4}$ [499 $\left.\rightarrow 353 \rightarrow 191\right]: 173$ (100), 171 (51.6), 127 (99.2), 111 (35.5), 109 (39.6)

$\mathrm{MS}^{2}$ [625]: 474 (16.1), 473 (100), 293 (8.6)

$\mathrm{MS}^{3}$ [625 $\rightarrow 473$ ]: 342 (8.7), 341 (100), 293 (51.1), 233 (22.9), 179 (20.7)

$\mathrm{MS}^{4}$ [625 $\left.\rightarrow 473 \rightarrow 341\right]: 239$ (46.4), 179 (100), 164 (21.9)

MS $^{2}$ [687]:643 (15.9), 601 (100), 597 (40.4), 557 (61.4), 437 (39.6)

$\mathrm{MS}^{3}$ [687 $\left.\rightarrow 601\right]: 557$ (65.6), 515 (15.0), 395 (100), 353 (31.1)

MS $^{4}[687 \rightarrow 601 \rightarrow 395]$ : $233(100)$

$\mathrm{MS}^{2}$ [515]: 354 (17.7), 353 (100), 191 (8.2), 179 (7.3), 173 (12.1)

$\mathrm{MS}^{3}$ [515 $\rightarrow 353$ ]: 191 (68.8), 179 (73.3), 173 (100), 135 (9.3)

$\mathrm{MS}^{4}[515 \rightarrow 353 \rightarrow 173]: 155(100), 111$ (75.6)

$\mathrm{MS}^{2}$ [499]: 338 (12.5), 337 (100), 335 (2.96), 173 (27.0)

$\mathrm{MS}^{3}$ [499 $\rightarrow 337$ ]: $173(100), 163(43.1)$

$\mathrm{MS}^{4}$ [499 $\rightarrow 337 \rightarrow 173$ ]: $111(100), 93$ (31.3)

MS $^{2}$ [497]: 337 (75.0), 335 (82.1), 255 (20.9), 179 (100), 173 (32.5)

$\mathrm{MS}^{3}$ [497 $\left.\rightarrow 179\right]: 136(2.6), 135(100)$

$\mathrm{MS}^{2}$ [529]: 368 (16.2), 367 (100), 179 (11.2), 161 (9.5)

$\mathrm{MS}^{3}$ [529 $\rightarrow$ 367]: 179 (100); 173 (23.4), 161 (54.9), 135 (79.8)

MS $^{4}$ [529 $\rightarrow 367 \rightarrow 179$ ]: 135 (100)

MS $^{2}$ [497]: 353 (45.7), 337 (29.6), 335 (63.5), 211 (27.3), 179 (100)
3,5-O-Dicaffeoylquinic acid

Total aerial parts Leaves

Stems

Malonyl-1,4-O-Dicaffeoylquinic acid

Stems

Malonyl-3,4-0-dicaffeoylquinic acid

Total aerial parts Leaves

4,5-O-Dicaffeoylquinic acid

Leaves

Malonyl-1,5-0-dicaffeoylquinic acid

Stems

Apigenin-7-O-glucuronide

Total aerial parts

Leaves

Stems

3-0-p-coumaroyl-4-0-caffeoylquinic acid

Leaves

Malonyl-4,5-0-dicaffeoylquinic acid

Total aerial parts Leaves

3-0-Coumaroyl-5-O-caffeoylquinic acid

Total aerial parts

3-O-Caffeoyl-5-O-coumaroylquinic acid

Total aerial parts

Caffeic acid derivative

Total aerial parts Stems

O-Dimalonyl-O-dicaffeoylquinic acid

Stems

1,4-O-Dicaffeoylquinic acid

Total aerial parts

4-O-p-coumaroyl-5-O-caffeoylquinic acid

Leaves

Dicaffeoylshikimic acid

Total aerial parts

Caffeic acid derivative

Total aerial parts

Dicaffeoylshikimic acid

Leaves

(continued on next page) 


\begin{tabular}{|c|c|c|c|c|c|c|}
\hline No. & $t_{R}(\min )$ & $\mathrm{UV} \lambda_{\max }(\mathrm{nm})$ & {$[\mathrm{M}-\mathrm{H}]^{-} m / z$} & HPLC-DAD-ESI-MS ${ }^{n} \mathrm{~m} / z$ (\% base peak) & Assigned identity & Morphological part \\
\hline & & & & $\begin{array}{l}\mathrm{MS}^{3}[497 \rightarrow 335]: 179(100), 161(48.6), 135(24.1) \\
\mathrm{MS}^{3}[499 \rightarrow 173]: 136(14.2), 135(100)\end{array}$ & & Stems \\
\hline 36 & 23.4 & - & 497 & $\begin{array}{l}\mathrm{MS}^{2}[497]: 335(22.5), 317(48.8), 273(100), 255(36.0), 211(46.2), 179(35.1), 161(32.9) \\
\mathrm{MS}^{3}[497 \rightarrow 273]: 255(49.8), 229(100), 211(82.0), 179(48.0), 159(50.6) \\
\mathrm{MS}^{4}[497 \rightarrow 273 \rightarrow 229]: 168(100)\end{array}$ & Unknown & Total aerial parts \\
\hline 37 & 23.9 & 265 & 497 & $\begin{array}{l}\mathrm{MS}^{2} \text { [497]: } 351(28.8), 317(100), 291(34.8), 273(83.1), 255(43.0), 231(32.1), 211 \text { (44.8), } 179 \text { (50.6) } \\
\left.\mathrm{MS}^{3} \text { [497 } \rightarrow 317\right]: 274(65.6), 256(37.1), 186(100) \\
\mathrm{MS}^{4}[497 \rightarrow 273 \rightarrow 229]: 168(100)\end{array}$ & Unknown & $\begin{array}{l}\text { Leaves } \\
\text { Stems }\end{array}$ \\
\hline 38 & 25.1 & - & 585 & $\begin{array}{l}\mathrm{MS}^{2}[585]: 543(28.5), 541(100), 395(37.7), 379(14.5) \\
\mathrm{MS}^{3}[585 \rightarrow 541]: 396(17.9), 395(78.7), 379(100) \\
\mathrm{MS}^{4}[585 \rightarrow 541 \rightarrow 379]: 233(100), 229(18.1), 173(26.7), 163(56.9)\end{array}$ & Caffeoyl-O-(malonyl)-O-coumaroylquinic acid & Leaves \\
\hline 39 & 26.1 & 216,287 & 341 & $\begin{array}{l}\operatorname{MS}^{2}[341]: 327(17.1), 326(100) \\
\operatorname{MS}^{3}[341 \rightarrow 326]: 311(100) \\
\operatorname{MS}^{4}[341 \rightarrow 326 \rightarrow 311]: 283(100), 281(25.6), 267(46.4)\end{array}$ & Dimethoxylflavanone & Stems \\
\hline 40 & 26.8 & - & 425 & $\begin{array}{l}\mathrm{MS}^{2}[425]: 179(100), 135(35.9) \\
\mathrm{MS}^{3}[425 \rightarrow 179]: 135(100)\end{array}$ & Caffeic acid derivative & $\begin{array}{l}\text { Total aerial parts } \\
\text { Leaves } \\
\text { Stems }\end{array}$ \\
\hline 41 & 28.2 & - & 445 & $\begin{array}{l}\operatorname{MS}^{2}[445]: 179(100), 135(64.0) \\
\operatorname{MS}^{3}[445 \rightarrow 179]: 135(100)\end{array}$ & Caffeic acid derivative & Leaves \\
\hline 42 & 29.1 & 244,327 & 557 & $\begin{array}{l}\mathrm{MS}^{2}[557]: 396(14.1), 395(100), 233(5.9) \\
\mathrm{MS}^{3}[557 \rightarrow 395]: 234(12.6), 233(100), 173(81.5) \\
\mathrm{MS}^{4}[557 \rightarrow 395 \rightarrow 233]: 173(100)\end{array}$ & Acetyldicaffeoylquinic acid & $\begin{array}{l}\text { Total aerial parts } \\
\text { Leaves } \\
\text { Stems }\end{array}$ \\
\hline $43^{*}$ & 29.2 & $245,291,327$ & 677 & $\begin{array}{l}\mathrm{MS}^{2} \text { [677]: } 516(23.2), 515(100), 353(13.8) \\
\mathrm{MS}^{3}[677 \rightarrow 515]: 353(100), 335(8.9), 191(22.6), 179(23.8), 173(59.7) \\
\mathrm{MS}^{4}[677 \rightarrow 515 \rightarrow 353]: 191(39.6), 179(74.6), 173(100), 135(16.5)\end{array}$ & 3,4,5-Tri-O-caffeoylquinic acid & $\begin{array}{l}\text { Total aerial parts } \\
\text { Leaves } \\
\text { Stems }\end{array}$ \\
\hline 44 & 33.0 & 249 & 707 & $\begin{array}{l}\mathrm{MS}^{2} \text { [707]: } 675(100), 545(92.0), 353(45.1), 513(32.6) \\
\left.\mathrm{MS}^{3} \text { [707 } \rightarrow 675\right]: 514(26.1), 513(100) \\
\mathrm{MS}^{4}[707 \rightarrow 675 \rightarrow 513]: 495(25.1), 339(100)\end{array}$ & Unknown & Leaves \\
\hline 45 & 33.2 & - & 327 & $\begin{array}{l}\mathrm{MS}^{2}[327]: 325(19.1), 291(40.5), 229(100), 211(64.5), 209(20.9), 171(56.7) \\
\mathrm{MS}^{3}[327 \rightarrow 229]: 227(46.7), 211(100), 165(95.4), 125(66.8) \\
\mathrm{MS}^{4}[327 \rightarrow 229 \rightarrow 211]: 135(100)\end{array}$ & Unknown & $\begin{array}{l}\text { Total aerial parts } \\
\text { Stems }\end{array}$ \\
\hline 46 & 34.0 & - & 583 & $\begin{array}{l}\mathrm{MS}^{2}[583]: 421(100), 259(6.8) \\
\mathrm{MS}^{3}[583 \rightarrow 421]: 259(100), 173(76.9) \\
\mathrm{MS}^{4}[583 \rightarrow 421 \rightarrow 259]: 173(100)\end{array}$ & Lamiridosins-di-O-hexoside & $\begin{array}{l}\text { Total aerial parts } \\
\text { Stems }\end{array}$ \\
\hline $47^{*}$ & 34.2 & 250,332 & 269 & $\begin{array}{l}\mathrm{MS}^{2} \text { [269]: } 227 \text { (54.4), } 226(38.8), 225 \text { (100), } 201 \text { (71.1), } 151(43.2), 149(61.0) \\
\left.\operatorname{MS}^{3} \text { [269 } \rightarrow 225\right]: 181(100)\end{array}$ & Apigenin & Leaves \\
\hline 48 & 34.7 & - & 659 & $\begin{array}{l}\mathrm{MS}^{2} \text { [659]: } 498(21.1), 497(100), 479(20.2), 453(14.4), 335(16.8) \\
\mathrm{MS}^{3}[659 \rightarrow 497]: 453(49.3), 335(100), 317(65.4), 179(51.1) \\
\mathrm{MS}^{4}[659 \rightarrow 497 \rightarrow 335]: 179(100), 135(67.9), 109(64.8)\end{array}$ & Tri-O-caffeoylshikimic acid & $\begin{array}{l}\text { Total aerial parts } \\
\text { Leaves }\end{array}$ \\
\hline 49 & 37.7 & - & 287 & $\mathrm{MS}^{2}$ [287]: 269 (100), 241 (57.4), 239 (41.4), 171 (39.4), 155 (61.9), 127 (37.2) & Unkown & Stems \\
\hline 50 & 38.1 & - & 599 & $\begin{array}{l}\mathrm{MS}^{2}[599]: 438(18.5), 437(100) \\
\mathrm{MS}^{3}[599 \rightarrow 437]: 275(100), 173(69.5) \\
\mathrm{MS}^{4}[599 \rightarrow 437 \rightarrow 275]: 173(100)\end{array}$ & Unknown & $\begin{array}{l}\text { Leaves } \\
\text { Stems }\end{array}$ \\
\hline
\end{tabular}


Flavonoids

Quercetin

R: Hexoside<smiles>[R20]c1cc(O)c2c(=O)c(OCC)c(-c3ccc(O)cc3)oc2c1</smiles>

Kaempferol

$\mathbf{R}_{1} / \mathbf{R}_{2}$ : Hexoside<smiles>O=c1cc(-c2ccc(O)cc2)oc2cc([O-])cc(O)c12</smiles>

Apigenin

Non-Flavonoids<smiles>O=C(O)C1(O)CC(O)C(O)C(O)C1[R]O</smiles>

Quinic acid

Mono-, di- and tri- $O$ isomers

R: Caffeic acid Malonic acid Coumaric
Hexoside<smiles>O=C(O)/C=C/c1ccc(O)cc1</smiles>

Coumaric acid<smiles>[R]OC1C=C(C(=O)O)CC([R6])C1O</smiles>

Shikimic acid

Di- and tri- $O$ isomers

R: Caffeic acid<smiles>O=C(O)C1OC(O)C(O)C(O)C1O</smiles>

Glucuronic acid<smiles>O=C(O)/C=C/c1ccc(O)c(O)c1</smiles>

Caffeic acid

R: Hexoside Rhamnoside<smiles>CC(=O)C1=COC(O)C2C1C(O)C(O)C2(C)O</smiles>

Lamiridosins dihexoside $\mathbf{R}=$ Hexoside

Fig. 2. Chemical structures of the main classes of compounds found in the methanolic extracts of Helichrysum obconicum.

no more $\mathrm{MS}^{n}$ data available, compound $\mathbf{4 2}$ was tentatively characterised as acetyldicaffeoylquinic acid.

\subsubsection{Malonylcaffeoylquinic acid}

Five caffeoylquinic acid derivatives containing a malonyl group in their structures were found.

Compounds 20 ( $\left.t_{R}=13.9 \mathrm{~min}\right), \mathbf{2 1}\left(t_{R}=14.5 \mathrm{~min}\right), \mathbf{2 3}\left(t_{R}=15.0\right.$ $\min ), 26\left(t_{R}=17.0 \mathrm{~min}\right)$ and $\mathbf{3 0}\left(t_{R}=19.6 \mathrm{~min}\right)$ displayed a $[\mathrm{M}-\mathrm{H}]^{-}$ ion at $m / z 601$ and gave characteristic fragment ions of malonyl $[\mathrm{M}-\mathrm{H}-44]^{-}$at $m / z 557$ and $[\mathrm{M}-\mathrm{H}-86]^{-}$at $m / z 515$ (Zhang, Shi, Qu, \& Cheng, 2007). However, their $\mathrm{MS}^{n}$ fragment ions intensities were different and it was possible to characterise them by comparison with literature data.

Compounds 20, 21 and 26 have been detected in our previous work with another Helichrysum species (Gouveia \& Castilho, 2009) under the same experimental conditions and were identified as malonyl-1,4-O-dicaffeoylquinic acid (compound 20), malonyl3,4-O-dicaffeoylquinic acid (compound 21 ) and malonyl-4,5-dicaffeoylquinic acid (compound 26).

$\mathrm{MS}^{n}$ fragmentation pattern of compound $\mathbf{2 3}$ was different from the isomers described above. MS ${ }^{2}$ fragmentation of the ion at $m / z$ 601 gave a fragment ion at $m / z 515$, as base peak, which represents the loss of $86 \mathrm{Da}$. The non observation of a $206 \mathrm{Da}$ loss in the MS ${ }^{2}$ spectrum reveals that the malonyl group is directly linked to the quinic acid structure. The dicaffeoylquinic acid residue $(\mathrm{m} / z 515)$ was submitted to further fragmentation and was characterised based on Clifford's hierarchical key (Clifford et al., 2005). The $\mathrm{MS}^{2}$ ions at $\mathrm{m} / \mathrm{z} 335$ and 179 were weak (ca. $1 \%$ and $0.71 \%$ of base peak, respectively) which matches the 1,5-O-dicaffeoylquinic acid structure, as observed for compound 18. Therefore, compound 23 was characterised as (malonyl)-1,5-O-diCQA.

Compound 30 displayed a $[\mathrm{M}-\mathrm{H}]^{-}$ion at $m / z$ 687. The $\mathrm{MS}^{2}$ spectrum gave several fragment ions at $m / z 643,557,437$ and, as base peak, a fragment ion at $m / z 601$. The occurrence of fragments at $m / z 643$ and 601 can be associated to the loss of 44 and $86 \mathrm{Da}$, respectively, which is characteristic of malonyl groups.

$\mathrm{MS}^{3}$ fragmentation of the ion at $m / z 601$ exhibited a fragment ion at $m / z 395$ (loss of $206 \mathrm{Da}$ ) as base peak. This type of fragmentation was described above for dicaffeoylquinic acids with malonyl moieties linked to one of the caffeoyl groups.

In compound 30, one of the malonyl groups is directly attached to the quinic acid structure and the other is linked to a caffeoyl group. Still, with no further $\mathrm{MS}^{n}$ information, the exact location of the substituent groups in the quinic acid structure is difficult to establish. So, compound $\mathbf{3 0}$ was characterised as $\mathbf{O}$-dimalonyl$O$-dicaffeoylquinic acid.

\subsubsection{Coumaroylquinic acid}

Amongst the above identified quinic acid derivatives, some isomers with a coumaroyl group on their structures could be found.

Compound $\mathbf{1 1}\left(t_{R}=8.3 \mathrm{~min}\right)$ showed a $[\mathrm{M}-\mathrm{H}]^{-}$ion at $\mathrm{m} / \mathrm{z} 337$ and was identified as 5-O-p-coumaroylquinic acid. This identification was achieved by comparison of the main fragment ions formed in the $\mathrm{MS}^{n}$ fragmentation experiments with those described in literature data (Clifford, Johnston, Knight, \& Kuhnert, 2003). This compound was only detected in the leaves extract.

Four compounds $(\mathbf{2 5}, \mathbf{2 7}, \mathbf{2 8}$ and $\mathbf{3 2})$ with a deprotonated molecular ion, $[\mathrm{M}-\mathrm{H}]^{-}$, at $m / z 499$ were found. They all showed 
close retention times which suggests a structural similarity. They were characterised based on the differences found on their $\mathrm{MS}^{n}$ spectra.

For compounds $25\left(t_{R}=16.6 \mathrm{~min}\right)$ and $28\left(t_{R}=17.8 \mathrm{~min}\right)$ the $\mathrm{MS}^{2}$ fragmentation of the MS ${ }^{1}$ ion at $\mathrm{m} / z 499$ showed the loss of a 146 Da residue (coumaroyl group) giving a caffeoylquinic acid fragment ion at $m / z 353$, as base peak.

For compound $\mathbf{2 5}$ the fragmentation of the ion at $m / z 353$ gave fragment ions at $m / z 173$ (base peak), 179 (ca. 50.0\% of base peak), 135 (ca. 30.0\% of base peak) and 191 (ca. 8\% of base peak). The occurrence of a base peak ion at $m / z 173$ indicates the presence of a quinic acid substituted at position 4-OH (Clifford et al., 2005). The exact location of the coumaroyl group is difficult to establish, but comparing our previously identification of coumaroycaffeoylquinic acid isomers and taking into account the results presented by Clifford, Marks, Knight, and Kuhnert (2006) compound 25 was identified as 3-O-p-coumaroyl-4-O-caffeoylquinic acid.

The [quinic acid-H] ${ }^{-}$ion at $m / z 191$ appeared in the compound $28 \mathrm{MS}^{3}$ spectrum as the base peak. Based on the main fragments detected on $\mathrm{MS}^{n}$ fragmentation and in literature data (Clifford et al., 2006), compound 28 was identified as 3-O-caffeoyl-5-O-pcoumaroylquinic acid.

The other two isomers, compounds $27\left(t_{R}=17.5 \mathrm{~min}\right)$ and $\mathbf{3 2}$ $\left(t_{R}=21.0 \mathrm{~min}\right)$, lost first the caffeoyl residue $(162 \mathrm{Da})$ in the $\mathrm{MS}^{2}$ fragmentation, originating a fragment ion at $m / z 337$. MS ${ }^{n}$ analysis of this ion revealed a coumaroylquinic acid structure. Compound 27 gave a $\mathrm{MS}^{3}$ fragment ion at $\mathrm{m} / z 163$ and compound 32 a MS ${ }^{3}$ fragment ion at $m / z 173$ as MS $^{3}$ spectra base peaks.

Based on Clifford et al. (2006) $\mathrm{MS}^{n}$ studies with this class of compounds it was possible to characterise compound 27 as 3-Op-coumaroyl,5-O-caffeoylquinic acid and compound 32 as $4-O-p$ coumaroyl,5-O-caffeoylquinic acid.

Compound $38\left(t_{R}=25.1 \mathrm{~min}\right)$ displayed a $[\mathrm{M}-\mathrm{H}]^{-}$ion at $\mathrm{m} / z$ 585 and was only detected in the leaves extract. MS $^{2}$ fragmentation of this ion gave a fragment ion at $\mathrm{m} / \mathrm{z} 541$ due to the loss of $44 \mathrm{Da}$, which corresponds to a dicarboxylation from a dicarboxylic acid. Additionally, another low intensity $\mathrm{MS}^{2}$ ion at $\mathrm{m} / \mathrm{z} 499$ was detected, which corresponds to the loss of 86 Da residue indicating a malonyl moiety. More precisely, this ion at $\mathrm{m} / \mathrm{z} 499$ can be assigned to a caffeoylcoumaroylquinic acid.

The $\mathrm{MS}^{3}$ spectrum showed two main fragment ions at $\mathrm{m} / z 379$ (base peak) and $\mathrm{m} / \mathrm{z} 395$ (ca. $94 \%$ of base peak) and were assigned to the loss of caffeoyl (162 Da) and coumaroyl (146 Da) moieties, respectively.

The subsequent fragmentation of these two ions led to a fragment ion at $m / z 233$ that was reported before as being acetylquinic acid (Gouveia \& Castilho, 2009).

The loss the caffeoyl group (162 Da) to form the base peak in the $\mathrm{MS}^{3}$ spectrum indicates that the malonyl group is linked to the coumaroyl group.

The exact location of the substituent groups in the quinic acid structure is difficult to establish based on the available $\mathrm{MS}^{n}$ data. Thus, compound $\mathbf{3 8}$ was assigned as caffeoyl-O-(malonyl)-Ocoumaroylquinic acid.

\subsubsection{Caffeoylshikimic acids}

Two compounds at a retention times of $21.1 \mathrm{~min}$ (compound 33) and $21.8 \mathrm{~min}$ (compound 35) showed a $[\mathrm{M}-\mathrm{H}]^{-}$ion at $\mathrm{m} / \mathrm{z}$ 497. Further $\mathrm{MS}^{n}$ fragmentation of the deprotonated molecular ion was identical for both compounds.

Under $\mathrm{MS}^{2}$ fragmentation, this ion easily lost $318 \mathrm{Da}$ to form a fragment ion at $m / z 179$, as base peak in the MS ${ }^{2}$ spectrum, which suggests the presence of a caffeic acid. The caffeic acid moiety was confirmed with the MS ${ }^{4}$ ions at $m / z 161$ and 135 formed from the dissociation the [caffeic acid-H] $]^{-}$ion, caused by the losses of $\mathrm{H}_{2} \mathrm{O}$ and $\mathrm{CO}_{2}$, respectively (Scheme 1 ).
Also in the $\mathrm{MS}^{2}$ spectrum, a strong fragment ion at $m / z 335$ was observed indicating the loss of $162 \mathrm{Da}$ from the deprotonated molecular ion indicating the presence of a caffeic acid residue. $\mathrm{MS}^{n}$ fragmentation of the ion at $\mathrm{m} / z 335$ showed the [caffeic acid-H $]^{-}$ion at $m / z 179$ and its dissociation fragment ions.

The fragmentation pattern observed for the ion at $m / z 335$ has been previously described in literature for a caffeoylshikimic acid (Fang, Yu, \& Prior, 2002; Hokkanen, Mattila, Jaakola, PirttilaÎ, \& Tolonen, 2009).

Therefore, based on these MS ${ }^{n}$ data, compounds 33 and 35 were characterised as having two caffeoyl groups and were characterised as dicaffeoylshikimic acids.

Compound 33 was detected only on the total aerial parts extract while compound $\mathbf{3 5}$ could be detected in the leaves and stems extracts of $H$. obconicum.

Compound $48\left(t_{R}=34.9 \mathrm{~min}\right)$ showed a $[\mathrm{M}-\mathrm{H}]^{-}$ion at $m / z 659$ which in $\mathrm{MS}^{2}$ fragmentation formed a fragment ion at $m / z$ 497, representing the loss of a 162 Da residue. $\mathrm{MS}^{n}$ fragmentation of the ion at $m / z 497$ was identical to that found for compounds $\mathbf{3 3}$ and $\mathbf{3 5}$, with main fragment ions at $m / z 335,179,161$ and 135 . The caffeoyl groups must be linked to each of the hydroxyl groups of shikimic acid, since fragment ions related to combined hexoside groups were not detected. Thus, compound $\mathbf{4 8}$ was characterised as a tricaffeoylshikimic acid.

Scheme 1 shows the proposed structures for the main fragment ions observed for this tricaffeoylshikimic acid derivative and the $\mathrm{MS}^{n}$ spectra for compound $\mathbf{4 8}$ are represented in Fig. 3. Due to the low absorption coefficient of shikimic acid, their UV spectra have not been properly observed.

To our knowledge, this was the first time that shikimic acid derivatives were identified in Helichrysum species and they could be found in all extracts of $H$. obconicum.

\subsubsection{Caffeic acid derivatives}

Compound $1\left(t_{R}=2.7 \mathrm{~min}\right)$ gave a $[\mathrm{M}-\mathrm{H}]^{-}$ion at $\mathrm{m} / \mathrm{z} 487$ and was found in the stems of $\mathrm{H}$. obconicum. Its $\mathrm{MS}^{2}$ fragmentation showed the loss of 146 Da resulting in a fragment ion at $\mathrm{m} / \mathrm{z} 341$. The $\mathrm{MS}^{3}$ spectrum of this ion showed the loss of a 162 Da residue, resulting in a fragment ion at $m / z 179$ which indicates the presence of a caffeic acid derivative. The two groups linked to the caffeic acid were identified as sugar unites, rhamnoside (146 Da) and a hexoside (162 Da), rather than hydroxycinnamic acids moieties due to the low retention time of compound 1. Therefore, compound 1 was characterised as caffeic acid-O-rhamnoside- $O$-hexoside.

Compound $2\left(t_{R}=2.9 \mathrm{~min}\right)$ originated a $[\mathrm{M}-\mathrm{H}]^{-}$ion at $\mathrm{m} / z 683$ as base peak and also a strong MS ${ }^{1}$ ion at $m / z 341$. MS ${ }^{2}$ fragmentation of the ion at $m / z 683$ gave a fragment ion at $m / z 341$ confirming that the ion at $m / z 683$ represents a dimer of the ion at $m / z 341$. Fragmentation of this ion $(m / z 341)$ gave a fragment ion at $m / z 179$, due to the loss of $162 \mathrm{Da}$ (hexoside residue), which point to a caffeic acid derivative. Thus, compound $\mathbf{2}$ was assigned as a dimer of caffeic acid $O$-hexoside and was only found in the total aerial parts extract.

Compounds $\mathbf{9}\left(t_{R}=7.3 \mathrm{~min}\right)$ and $\mathbf{1 0}\left(t_{R}=7.7 \mathrm{~min}\right)$ displayed the same $[\mathrm{M}-\mathrm{H}]^{-}$ion at $m / z$ 533. They presented a similar $\mathrm{MS}^{n}$ fragmentation pattern but compound $\mathbf{9}$ was detected in the total aerial parts and compound $\mathbf{1 0}$ in the leaves extract.

Their $\mathrm{MS}^{2}$ spectra showed a fragment ion at $m / z 371$, as base peak, due to the loss of 162 Da corresponding to a hexoside moiety.

The sequential $\mathrm{MS}^{n}$ fragmentation and the detection of fragment ions at $m / z 353$ and 179 led to the identification of a caffeic acid residue. With no further information available compounds $\mathbf{9}$ and $\mathbf{1 0}$ were assigned as caffeic acid- $O$-hexoside derivatives.

Five other compounds 15, 29, 34, 40 and 41 were detected and also characterised as caffeic acid derivatives. They all exhibited different $[\mathrm{M}-\mathrm{H}]^{-}$ions and distinct $\mathrm{MS}^{n}$ fragmentation behaviour but 


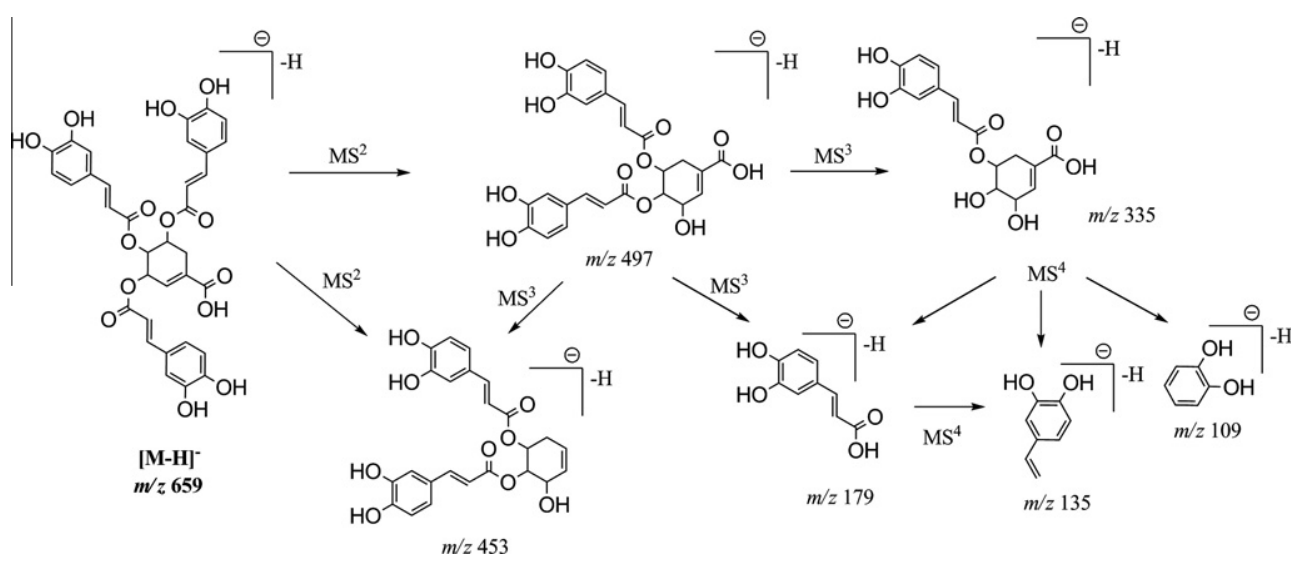

Scheme 1. Proposed fragmentation pathway for compound 48 - Tri-O-caffeoylshikimic acid.

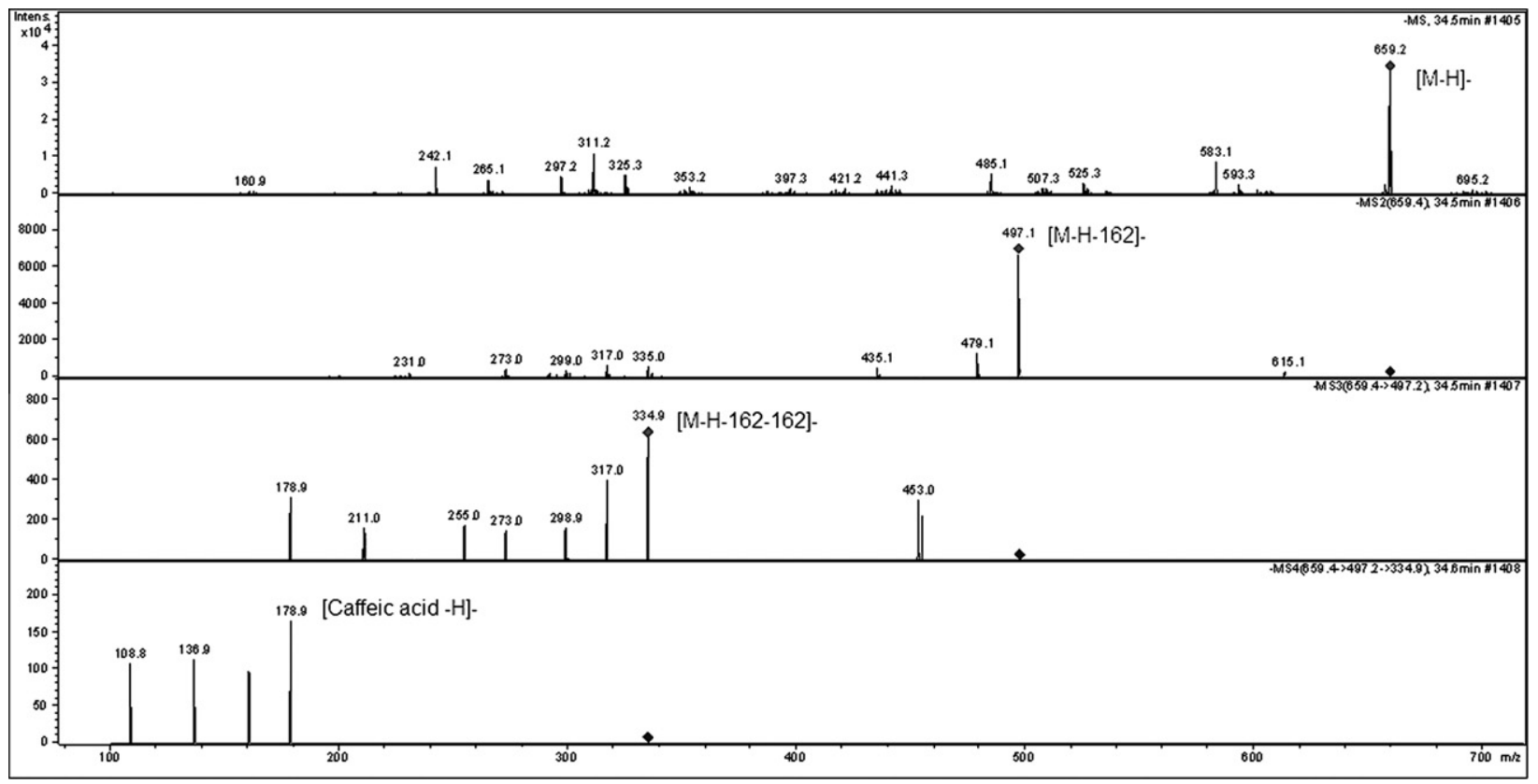

Fig. 3. ESI-MS ${ }^{n}$ negative mode of compound 48. Sequential fragmentation, $\mathrm{MS}^{n}(n=2-4)$, of the ion at $m / z 659$.

they all had in common a fragment ion at $m / z 179$ that corresponds to a deprotonated caffeic acid ion, [caffeic acid-H] ${ }^{-}$.

Compound $15\left(t_{R}=11.4 \mathrm{~min}\right)$ exhibited a $[\mathrm{M}-\mathrm{H}]^{-}$ion at $\mathrm{m} / z 415$ and was found in the total aerial parts extract. The $\mathrm{MS}^{2}$ spectrum showed a fragment ion at $\mathrm{m} / \mathrm{z} 179$ due to the loss of a $236 \mathrm{Da}$ residue but its nature could not be identified.

Compound $29\left(t_{R}=18.3 \mathrm{~min}\right)$ showed a $[\mathrm{M}-\mathrm{H}]^{-}$ion at $m / z 625$ which easily lost a $152 \mathrm{Da}$ residue to form a fragment ion at $\mathrm{m} / \mathrm{z}$ 473. $\mathrm{MS}^{3}$ fragmentation of this ion gave a fragment ion at $\mathrm{m} / \mathrm{z}$ 341 due to the loss of 132 Da residue (could be a pentose or a tartaric acid moiety). The [caffeic acid-H] ${ }^{-}$ion was found to be the $\mathrm{MS}^{4}$ spectrum base peak. There were not observed more fragment ions useful in the identification of the substituent groups. This compound was detected in the total aerial parts and in the stems extract.

Compound $34\left(t_{R}=21.7 \mathrm{~min}\right)$ exhibited a $[\mathrm{M}-\mathrm{H}]^{-}$ion at $\mathrm{m} / \mathrm{z}$ 529. Its $\mathrm{MS}^{2}$ spectrum showed a fragment ion at $\mathrm{m} / \mathrm{z} 367$ (loss of $162 \mathrm{Da}$ ). The $\mathrm{m} / \mathrm{z} 367$ ion indicates the presence of a feruloylquinic acid structure, but it was not possible to detect more fragments to support this idea. The tentatively characterisation of this compound as a caffeic acid derivative results from the observation of a $\mathrm{MS}^{3}$ ion at $m / z 179$.

Compound $40\left(t_{R}=26.8 \mathrm{~min}\right)$ was detected in all extracts and gave a $[\mathrm{M}-\mathrm{H}]^{-}$ion at $\mathrm{m} / \mathrm{z} 425$ MS $^{2}$ fragmentation showed a loss of $246 \mathrm{Da}$ to form a fragment ion at $m / z 179$.

Compound $41\left(t_{R}=28.2 \mathrm{~min}\right)$ displayed a $[\mathrm{M}-\mathrm{H}]^{-}$ion at $\mathrm{m} / \mathrm{z}$ 445. Its $\mathrm{MS}^{2}$ spectrum showed a fragment ion at $\mathrm{m} / z 179$ due to the loss of a 266 Da residue. Without any other information, the nature of the neutral losses of 246 Da (compound 40) and 266 (compound 41) was not possible to identify and fully characterise these two compounds.

\subsection{Flavonoids and lamarosinin-di-O-hexoside}

In addition to the phenolic acid derivatives described above, there were also identified a few flavonoid compounds in $\mathrm{H}$. obconicum. Almost all flavonoids were found in their glycoside form, containing one or more sugar units, and some were esterified with acyl groups. Free aglycones were found in trace amounts in some samples. 
The flavonoid fragment ions were labelled according to the nomenclature proposed by Ma, Li, Heuvel, and Claeys (1997). For free aglycones, the ${ }^{i, j} \mathrm{~A}^{-}$and ${ }^{i, j} \mathrm{~B}^{-}$labels correspond to ions containing intact $\mathrm{A}$ - and $\mathrm{B}$ - rings, respectively, in which $\mathrm{i}$ and $\mathrm{j}$ indicate de C-Ring bonds that have been broken. For conjugated aglycones, $Y_{0}^{-}$ is used to refer to the aglycone fragment $[\mathrm{M}-\mathrm{H} \text {-glycoside }]^{-}$. Compound $7\left(t_{R}=6.0 \mathrm{~min}\right)$ gave a $[\mathrm{M}-\mathrm{H}]^{-}$ion at $\mathrm{m} / \mathrm{z} 609$ and its $\mathrm{MS}^{2}$ fragmentation showed the aglycone ion, $Y_{0}^{-}$, at $m / z 285$ as base peak and also a fragment ion at $\mathrm{m} / \mathrm{z} 447$ (ca. $31.7 \%$ of base peak).

The aglycone ion was formed by the loss of a residue of $324 \mathrm{Da}$ composed of two hexoside moieties $(2 \times 162 \mathrm{Da})$. Since the aglycone radical ion was not detected in the $\mathrm{MS}^{2}$ spectrum and based on the rules reported by Ablajan et al. (2006) this compound was primarily characterised as a flavonoid-O-diglycoside.

The $\mathrm{MS}^{3}$ fragmentation of the ion at $\mathrm{m} / \mathrm{z} 285$ gave fragment ions at $\mathrm{m} / \mathrm{z} 213$ (95.5\% of base peak), 257 (46.8\% of base peak), 163 (56.0\% of base peak) and a fragment ion at $m / z 255$ as base peak. These retro-diels-Alder (RDA) fragments are consistent with those found for a standard solution of kaempferol (MS ${ }^{n}$ fragmentation data not shown).

For flavonols like kaempferol the 7-OH and 3-OH are the most common glycosylation positions; those compounds substituted at position 3-OH should present relative high intensity aglycone radical fragment sometimes higher than the $Y_{0}^{-}$ion (Cuyckens \& Claeys, 2005). Such pattern was not observed and the exact location of the hexosides moieties could not be established.

In the $\mathrm{MS}^{2}$ spectrum there were ions corresponding to the loss of a hexoside moiety (162 Da) at $\mathrm{m} / \mathrm{z} 447$ and a hexoside plus water moiety ( $180 \mathrm{Da}$ ) at $\mathrm{m} / \mathrm{z} 429$ and these fragments are associated to the break of a $(1 \rightarrow 2)$ interglycosidic linkage (Ferreres, Llorach, \& Gil-Izquierdo, 2004).

Thus, compound 7 was identified as kaempferol-O-( $1 \rightarrow 2)$ dihexoside and could only be detected in the leaves extract.

Compound $39\left(t_{R}=26.1 \mathrm{~min}\right)$ displayed a $[\mathrm{M}-\mathrm{H}]^{-}$ion at $\mathrm{m} / \mathrm{z}$ 341. Its UV profile (absorption maximum bands at 216 and $287 \mathrm{~nm}$ ) suggests a flavanone or a dihydrochalcone skeleton as proposed by Portet et al. (2008). Its $\mathrm{MS}^{2}$ and $\mathrm{MS}^{3}$ spectra showed, as base peak, fragment ions at $\mathrm{m} / \mathrm{z} 326$ and 311 (losses of $15 \mathrm{Da}$ ) due to the consecutive radical loss of two methyl groups indicating a methoxylated compound. The $\mathrm{MS}^{4}$ fragmentation of the ion at $\mathrm{m} /$ $z 311$ gave a fragment ion at $\mathrm{m} / z 283$ indicating the neutral loss of a CO molecule (28 Da).

The lack of an important loss of $\mathrm{H}_{2} \mathrm{O}$ points out a flavanone skeleton (Portet et al., 2008). The ion at $\mathrm{m} / \mathrm{z} 283$ did not present sufficient intensity to allow further fragmentation in order to fully characterise compound 39, in particular the position of the substitution groups in the flavanone skeleton. Thus, this compound was tentatively characterised as a dimethoxylflavanone.

Compound $12\left(t_{R}=8.4 \mathrm{~min}\right)$ showed a $[\mathrm{M}-\mathrm{H}]^{-}$ion at $m / z 567$ and its $\mathrm{MS}^{2}$ fragmentation formed a fragment ion at $m / z 341$ due to the loss of a 226 Da residue. Further fragmentation of the ion at $m / z$ 341 gave similar fragmentation behaviour to that found for compound 39. The nature of the $226 \mathrm{Da}$ residues could not be determined but it is clear that is must be a hydrophilic group given the low retention time of elution of this compound. Therefore, compound 12 was tentatively characterised as being a dimethoxylflavanone derivative.

Compound 12 was found in the total aerial parts and stems extracts while compound $\mathbf{3 9}$ was only detected in the stems extract.

Compound $14\left(t_{R}=10.1 \mathrm{~min}\right)$ displayed a $[\mathrm{M}-\mathrm{H}]^{-}$ion at $\mathrm{m} / \mathrm{z}$ 463. The $\mathrm{MS}^{2}$ spectrum revealed the loss of a hexoside residue (162 Da) giving the aglycone ion, $Y_{0}^{-}$, at $m / z 301$ as base peak.

Its $\mathrm{MS}^{n}$ fragmentation led to the detection of RDA fragment ions at $\mathrm{m} / \mathrm{z} 179\left(\left[^{1,2} \mathrm{~A}^{-}-\mathrm{H}\right]^{-}\right), 271\left(\left[\mathrm{M}-\mathrm{H}-\mathrm{CH}_{2} \mathrm{O}\right]^{-}\right), 255\left(\left[\mathrm{M}-\mathrm{H}-\mathrm{H}_{2}\right.\right.$ $\left.\mathrm{O}-\mathrm{CO}]^{-}\right)$and $151\left({ }^{1,2} \mathrm{~A}^{-}-\mathrm{CO}\right)$ which are characteristic ions of quercetin (Gouveia \& Castilho, 2009, 2010). Compound 14 was identified by comparison of $\mathrm{MS}^{n}$ and UV data (bands at $258 \mathrm{~nm}$ and $353 \mathrm{~nm}$ ) obtained for a standard solution of quercetin-3-O-glucoside.

This compound was only found in the leaves of H. obconicum, and it has been already reported in Helichrysum devium and Helichrysum melaleucum (Gouveia \& Castilho, 2009, 2010).

Compound $47\left(t_{R}=34.2 \mathrm{~min}\right)$ showed a $[\mathrm{M}-\mathrm{H}]^{-}$ion at $m / z 269$. Comparison of the main $\mathrm{MS}^{n}$ fragment ions such as those at $m / z$ $225\left(\left[\mathrm{M}-\mathrm{H}-\mathrm{CO}_{2}\right]^{-}\right), 201\left(\left[\mathrm{M}-\mathrm{H}-\mathrm{C}_{3} \mathrm{O}_{2}\right]^{-}\right), 151\left({ }^{1,3} \mathrm{~A}\right)$ and 149 $\left({ }^{1,4} \mathrm{~B}+2 \mathrm{H}\right)$, UV spectrum (bands at 250 and $332 \mathrm{~nm}$ ) and the HPLC retention time to those obtained for a standard solution allowed for the identification of compound $\mathbf{4 7}$ as apigenin. It was only

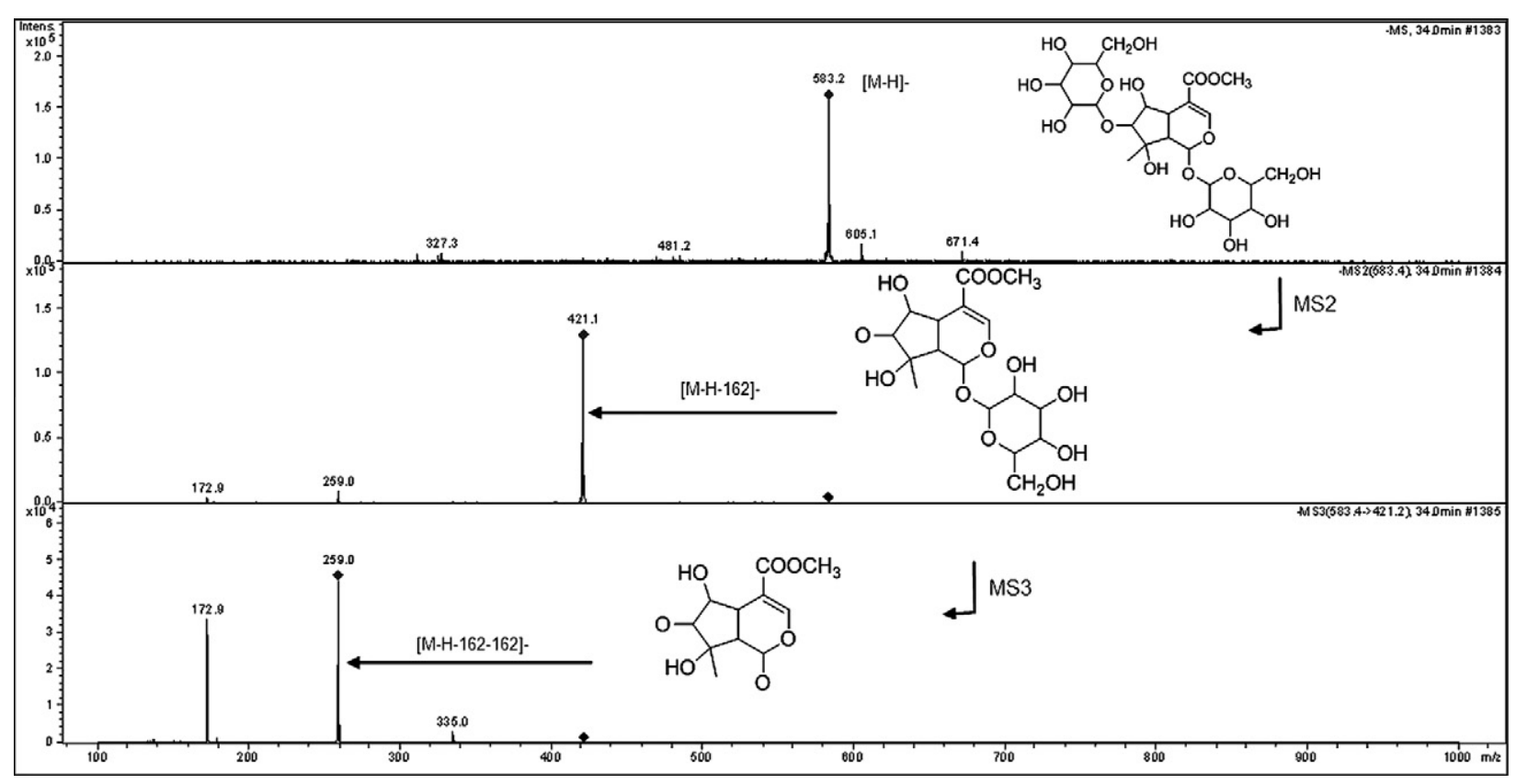

Fig. 4. ESI-MS ${ }^{n}$ negative mode of compound $\mathbf{4 6}$ - lamiridosins-di-O-hexoside. Sequential fragmentation, MS ${ }^{n}(n=2-3)$, of the ion at $m / z 583$. 
found in the leaves extract, although all extracts gave a substituted form of apigenin (compound 24).

Compound $24\left(t_{R}=15.7 \mathrm{~min}\right)$ gave a $[\mathrm{M}-\mathrm{H}]^{-}$ion at $m / z 445$ and subsequent fragmentation showed the loss of $176 \mathrm{Da}$, which corresponds to a glucuronic acid moiety. Fragmentation of the aglycone ion, $Y_{0}^{-}$, at $m / z 269$ gave fragment ions at $m / z 227,225,169,151$ and 149. This fragmentation behaviour is similar to that found for apigenin (compound 47). As it is known, for flavones, the most common substitution position is the 7-OH, therefore compound $\mathbf{2 4}$ was identified as apigenin-7-O-glucuronide. All morphological parts analysed of $H$. obconicum revealed the presence of this compound.

Compound $46\left(t_{R}=34.0 \mathrm{~min}\right)$ exhibited a $[\mathrm{M}-\mathrm{H}]^{-}$ion at $\mathrm{m} / \mathrm{z} 583$ and its $\mathrm{MS}^{2}$ spectrum gave a fragment ion at $\mathrm{m} / z 421$ due to the loss of 162 Da probably a hexoside residue. $\mathrm{MS}^{3}$ fragmentation of the ion at $m / z 421$ showed the loss of a 162 Da residue forming a fragment ion at $m / z 259$ assigned as the aglycone ion, $Y_{0}^{-}$. The aglycone ion under $\mathrm{MS}^{4}$ fragmentation displayed a fragment ion at $\mathrm{m} / \mathrm{z}$ 173 (Fig. 4). Comparing these results with literature data, the ion at $\mathrm{m} / \mathrm{z} 421$ was identified as lamalbid (Alipieva, Kokubun, Taskova, Evstatieva, \& Handjieva, 2007). Lamalbid belongs to the group of iridoids and is a hexoside of lamiridosins. Lamiridosins (aglycone part) is the name given to the two inseparable epimers (carbon 1 is a chiral carbon) (Zhang et al., 2009). Therefore, compound 46 was identified as lamiridosins-di-O-hexoside (Fig. 4).

This class of compounds consists in a large group of natural monoterpenes with taxonomic marker properties. More precisely, lamiridosins are known for its anti-HCV pp activity (Zhang et al., 2009).

\subsection{Unidentified compounds}

Two compounds with $[\mathrm{M}-\mathrm{H}]^{-}$ion at $m / z 497$ were observed at a retention time of 23.4 and $24.1 \mathrm{~min}$. However, their $\mathrm{MS}^{n}$ fragmentation behaviour was very distinct to that found for compounds 33 and 35, also with $[\mathrm{M}-\mathrm{H}]^{-}$ion at $m / z 497$.

$\mathrm{MS}^{2}$ fragmentation showed the loss of $224 \mathrm{Da}$ to form a fragment ion at $m / z$ 273. Further $\mathrm{MS}^{n}$ fragmentation gave fragment ions at $m / z 109$ and 168 . However, it was not possible to positively identify them.

Compound $44\left(t_{R}=33.0 \mathrm{~min}\right)$ showed a $[\mathrm{M}-\mathrm{H}]^{-}$ion at $m / z 707$ and in the $\mathrm{MS}^{3}$ spectrum the loss of 162 Da residue was observed, probably due to a hexoside residue. It was only found in the leaves extract.

Compound $49\left(t_{R}=37.7 \mathrm{~min}\right)$ showed a $[\mathrm{M}-\mathrm{H}]^{-}$ion at $\mathrm{m} / \mathrm{z} 287$ and its $\mathrm{MS}^{2}$ fragmentation gave a fragment ion at $\mathrm{m} / \mathrm{z} 269$ (loss of $18 \mathrm{Da}$, probably a $\mathrm{H}_{2} \mathrm{O}$ molecule). The ion at $\mathrm{m} / z 269$ is characteristic for apigenin (compound 47). However, the low intensity of this ion did not allowed for further fragmentation in order to fully characterise it and the UV spectrum did not provide any valid information; thus identification of compound 49, only found in the stems, was not achieved.

\section{Conclusion}

50 phenolic compounds were characterised in the different morphological parts of $H$. obconicum, based on their HPLC retention time, UV spectra and mass fragmentation behaviour.

Dicaffeoylquinic acids and specifically malonyl-dicaffeoylquinic acids are the main components of $H$. obconicum methanolic extracts. Other derivatives of the esters of caffeic acid and quinic acid or shikimic acids were also detected in minor amounts. Moreover, it was the first time, to our knowledge, that shikimic acid derivatives and iridoids hexosides were found in Helichrysum species.

The phenolic composition of $\mathrm{H}$. obconicum shared 20 of its 50 components with $H$. devium or $H$. melaleucum albeit in very different proportions. This conclusion is in good agreement with the fact that $H$. obconicum is used in the local traditional medicine with different purposes (stomachic diseases) of the two other endemic species, used for respiratory problems.

\section{Acknowledgements}

S. Gouveia is grateful to Fundação para a Ciência e Tecnologia (Portugal) for a Ph.D. Grant SFRH/BD/24227/2005.

The Mass Spectrometer used in this work is part of the Portuguese National Mass Spectrometry Network (Contract RNEMREDE/1508/REM/2005) and was purchased in the framework of the National Programme for Scientific Re-equipment, with funds from POCI 2010 (FEDER) and Fundação para a Ciência e a Tecnologia.

The authors wish to acknowledge the contribution of taxonomist Fátima Rocha in plant collection and identification.

\section{References}

Ablajan, K., Abliz, Z., Shang, X.-Y., He, J.-M., Zhang, R.-P., \& Shi, J.-G. (2006). Structural characterization of flavonol 3, 7-di-O-glycosides and determination of the glycosylation position by using negative ion electrospray ionization tandem mass spectrometry. Journal Mass Spectrometry, 41, 352-360.

Alipieva, K., Kokubun, T., Taskova, R., Evstatieva, L., \& Handjieva, N. (2007). LC-ESIMS analysis of iridoid glucosides in Lamium species. Biochemical Systematics and Ecology, 35, 17-22.

Al-Rehaily, A. J., Albishi, O. A., El-Olemy, M. M., \& Mossa, J. S. (2008). Flavonoids and terpenoids from Helichrysum forskahlii. Phytochemistry, 69, 1910-1914.

Carini, M., Aldini, G., Furlanetto, S., Stefani, R., \& Facino, R. M. (2001). LC coupled to ion-trap MS for the rapid screening and detection of polyphenol antioxidants from Helichrysum stoechas. Journal of Pharmaceutical and Biomedical Analysis, 24, 517-526.

Clifford, M. N., Johnston, K. L., Knight, S., \& Kuhnert, N. (2003). Hierarchical scheme for LC-MS ${ }^{n}$ identification of chlorogenic acids. Journal of Agricultural and Food Chemistry, 51, 2900-2911.

Clifford, M. N., Knight, S., \& Kuhnert, N. (2005). Discriminating between the six isomers of dicaffeoylquinic acid by LC-MS ${ }^{n}$. Journal of Agricultural and Food Chemistry, 53, 3821-3832.

Clifford, M. N., Marks, S., Knight, S., \& Kuhnert, N. (2006). Characterization by LC$\mathrm{MS}^{n}$ of four new classes of $p$-coumaric acid-containing diacyl chlorogenic acids in green coffee beans. Journal Agricultural Food Chemistry, 54, 4095-4101.

Cuyckens, F., \& Claeys, M. (2004). Mass spectrometry in the structural analysis of flavonoids. Journal of Mass Spectrometry, 39, 1-15.

Cuyckens, F., \& Claeys, M. (2005). Determination of the glycosylation site in flavonoid mono-O-glycosides by collision-induced dissociation of electrospraygenerated deprotonated and sodiated molecules. Journal Mass Spectrometry, 40, 364-372.

Fang, N., Yu, S., \& Prior, R. L. (2002). LC/MS/MS characterization of phenolic constituents in dried plums. Journal of Agricultural and Food Chemistry, 50, 3579-3585.

Ferreres, F., Llorach, R., \& Gil-Izquierdo, A. (2004). Characterization of the interglycosidic linkage in di-, tri-, tetra- and pentaglycosylated flavonoids and differentiation of positional isomers by liquid chromatography/electrospray ionization tandem mass spectrometry. Journal of Mass Spectrometry, 39, 312-321.

Gouveia, S. C., \& Castilho, P. C. (2009). Analysis of phenolic compounds from different morphological parts of Helichrysum devium by liquid chromatography with on-line UV and electrospray ionization mass spectrometric detection. Rapid Communications in Mass Spectrometry, 23, 3939-3953.

Gouveia, S. C., \& Castilho, P. C. (2010). Characterization of phenolic compounds in Helichrysum melaleucum by high-performance liquid chromatography with online ultraviolet and mass spectrometry detection. Rapid Communications in Mass Spectrometry, 24, 1851-1868.

Hokkanen, J., Mattila, S., Jaakola, L., Pirttilâิ, A. M., \& Tolonen, A. (2009). Identification of phenolic compounds from Lingonberry (Vaccinium vitis-idaea L.), Bilberry (Vaccinium myrtillus L.) and Hybrid Bilberry (Vaccinium $x$ intermedium Ruthe L.) leaves. Journal of Agricultural and Food Chemistry, 57, 9437-9447.

Ma, Y. L., Li, Q. M., Heuvel, H. V. d., \& Claeys, M. (1997). Characterization of flavone and flavonol aglycones by collision-induced dissociation tandem mass spectrometry. Rapid Communications in Mass Spectrometry, 11, 1357-1364.

Mabry, T. J., Markham, K. R., \& Thomas, M. B. (1970). The ultraviolet spectra of flavones and flavonols, isoflavones, dihydroxyflavonols. In The systematics identification of flavonoids. New York, NY: Springer-Verlag.

Mattila, P., \& Kumpulainen, J. (2002). Determination of free and total phenolic acids in plant-derived foods by HPLC with diode-array detection. Journal of Agricultural and Food Chemistry, 50, 3660-3667. 
Portet, B., Fabre, N., Rozenberg, R., Habib-Jiwan, J.-L., Moulis, C., \& QuetinLeclercq, J. (2008). Analysis of minor flavonoids in Piper hostmannianum var. Berbicense using liquid chromatography coupled with atmospheric pressure chemical ionization mass spectrometry. Journal of Chromatography A, 1210, 45-54.

Rivera, D., \& Obón, C. (1995). The ethnopharmacology of Madeira and Porto Santo Islands, a review. Journal of Ethnopharmacology, 46, 73-93.
Zhang, H., Rothwangl, K., Mesecar, A. D., Sabahi, A., Rong, L., \& Fong, H. H. S. (2009) Lamiridosins, Hepatitis C Virus entry inhibitors from Lamium album. Journal of Natural Products, 72, 2158-2162.

Zhang, Y., Shi, P., Qu, H., \& Cheng, Y. (2007). Characterization of phenolic compounds in Erigeron breviscapus by liquid chromatography coupled to electrospray ionization mass spectrometry. Rapid Communications in Mass Spectrometry, 21, 2971-2984. 